\title{
Towards Motion-Robust Magnetic Resonance Thermometry
}

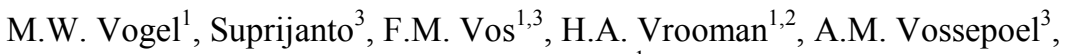 \\ P.M.T. Pattynama ${ }^{1}$ \\ ${ }^{1}$ Department of Radiology, Erasmus University Rotterdam, The Netherlands \\ mvogel@rdiag.fgg.eur.nl,.p.pattynama@inter.nl.net \\ ${ }^{2}$ Department of Medical Informatics, Erasmus University Rotterdam, The Netherlands \\ vrooman@mi.fgg.eur.nl \\ ${ }^{3}$ Department of Applied Physics, Delft University of Technology, The Netherlands \\ \{supri, frans, albert\}@ph.tn.tudelft.nl
}

\begin{abstract}
Magnetic Resonance Imaging allows for minimally invasive targeting and thermal ablation of tumors while monitoring the temperature distribution. Conventional MR thermometry procedures are hampered by either low accuracy or high sensitivity to motion artifacts due to the use of a reference temperature image. A new, dual-echo technique has been developed to obtain the temperature distribution within a single MR-acquisition. The acquired phase images were post-processed using noise filtering and advanced phase unwrapping, to obtain the two-dimensional temperature distribution. In vitro calibration experiments showed that the accuracy of our newly developed technique is similar to existing thermometry approaches. There was a good linear relationship $\left(r^{2}=0.99\right)$ between the measured phase difference and the recorded temperature up to at least $65^{\circ} \mathrm{C}$. The reproducibility of the temperature coefficient $(\Delta \varphi / \Delta T)$ was within $5 \%$. Real-time temperature mapping and solving susceptibility inhomogeneity are under investigation.
\end{abstract}

\section{Introduction}

On-going improvements in recognition and staging of tumors allow for early management of related morbidity. Tumor size is usually controlled by suppressive chemotherapy. Minimally invasive surgery may be especially beneficial in the early stage of cancer, when lesions are small. Thermal surgery may be an appropriate form of minimally invasive surgery since coagulated tissue allows for regeneration of healthy tissue.

The curative effect of (ablative) hyperthermia on soft tissue tumors has been known for over half a century. The resulting heat distribution, however, is difficult to predict. Due to the a priori unknown differences in thermal conductivity, diffusion and physiological cooling effects, the efficiency of hyperthermic applications is greatly reduced. Models for predicting the temperature distribution, especially in the higher temperature range used for ablative therapy, are not yet fully established [1]. With the availability of advanced imaging equipment, temperature changes during treatment can be visualized using ultrasound [2] or Magnetic Resonance Imaging 
(MRI) $[3,4]$. Use of temperature information allows for monitoring and controlling temperature related events $[5,6]$. Registering changes in temperature for specific pixels allows for precise thermal dose calculation [7].

MRI has been favored for monitoring local hyperthermia therapy, since it offers both target visualization and temperature sensitivity. Temperature sensitivity by MRI has been demonstrated for the apparent diffusion constant, the spin-lattice relaxation time $\left(\mathrm{T}_{1}\right)$, and the water proton resonance frequency shift. The frequency shift approach is accurate, but very sensitive to motion artifacts that may lead to corrupted temperature information.

The objective of this paper is to show that sufficiently accurate temperature information can be collected in a single MR-acquisition using a newly developed procedure, which may apply for thermometry purposes in vivo.

\section{Materials \& Methods}

There is a direct relationship between tissue temperature and proton (spin) mobility [8-10]. For diamagnetic materials, such as soft tissues in the human body, the moving electrons around the proton spin result in a small local magnetic field, opposing the applied field $\left(B_{0}\right)$. This shielding is temperature dependent. For water, the screening constant $\alpha$ shows a linear increase with increasing temperature over a broad range $\left(T \varepsilon\left[0,83^{\circ} \mathrm{C}\right]\right)[9,11]$.

\subsection{Conventional Phase Shift Thermometry}

The change in the local magnetic field causes a change in the Larmor frequency, resulting in phase differences in the acquired MR phase image. The measured phase $\Delta \phi$ can be used for calculating differences in temperature $\Delta T$, according to [12]:

$$
\Delta \varphi=\gamma \cdot \alpha \cdot B_{0} \cdot \Delta T \cdot t e
$$

where $\gamma$ is the gyromagnetic ratio, $\alpha$ the temperature coefficient, te the echo time, and $B_{0}$ the main magnetic field.

Measured phase is relative to the phase at initial temperature and therefore easily invalidated when voxels are displaced by patient breathing or patient movement. Recent studies have shown the feasibility of MR-controlled hyperthermia using the phase shift method, albeit in the absence of tissue motion [13]. In order to be able to employ the phase shift, the method should be robust for phase changes that result from motion.

\subsection{Dual-Echo Phase Shift Thermometry}

Because currently not all movement artifacts can be corrected for, we investigated the use of the phase that develops between two subsequent echoes for the measurement of 
temperature, instead of referencing to the static phase reference from a fixed geometry that was acquired prior to heating. The difference between two sampled phases for the same region yields, with a given period, a specific frequency for that region, which translates into local temperature differences.

We modified the standard spoiled gradient echo sequence to incorporate a second readout period. If two echoes are acquired successively using one excitation only, this yields:

$$
\begin{gathered}
\varphi_{1}-\varphi_{0}=\gamma \cdot \alpha \cdot B_{0} \cdot\left(T_{1}-T_{0}\right) \cdot t e_{1}, \\
\varphi_{2}-\varphi_{0}=\gamma \cdot \alpha \cdot B_{0} \cdot\left(T_{2}-T_{0}\right) \cdot t e_{2}
\end{gathered}
$$

Now if we assume that

$$
T_{1} \approx T_{2} \Rightarrow \Delta \varphi_{2-1}=k \cdot \Delta T \cdot\left(t e_{2}-t e_{1}\right),
$$

where $\mathrm{k}$ incorporates the constant terms, temperature relates directly to the phase evolution. When we take into account the surroundings of the object, for which we assume identical shielding and main magnetic field, we can normalize the evolution of phase to the pixels in the vicinity, rather than using base images.

Analysis of the local magnetic field in a chemically homogeneous sphere, shows that the macroscopic susceptibility is approximated by $H^{*}\left(1-2 *\left(\chi_{e}-\chi_{o}\right) / 3\right)$ [14]. At the microscopic level, we arrive at an expression for the local magnetic field that is only a function of the temperature dependent screening constant.

\subsection{Experiments}

To demonstrate the reproducibility of the conventional thermometric procedure and to compare the measured $\alpha$ with values in the literature, an experiment was carried out in which phase images from a phantom $(n=10)$ were acquired (Experiment 1$)$. The used phantom was a gel (5\% agar, Sigma; $95 \%$ distilled water) that was heated in a water bath up to $70^{\circ} \mathrm{C}$ and was put in a Styrofoam box. This setup was placed in the MR-scanner, where it was allowed to cool down to $30^{\circ} \mathrm{C}$ during continuous acquisition of MR images. In total, more than 100 time samples were acquired. MR hardware consisted of a GE CV/i $1.5 \mathrm{~T}$ scanner (slew rate $150 \mathrm{Tm}^{-1} \mathrm{~s}^{-1}$, gradient strength $40 * 10^{-3} \mathrm{Tm}^{-1}$, rise time $268 * 10^{-6} \mathrm{~s}$ ). Scanning was done using a conventional gradient echo scanning technique (Table 1). Gel temperature was simultaneously recorded using a fiber optic measurement device (Luxtron 790, Luxtron, Santa Clara, USA). The fibers were positioned in both the phantom and a reference gel.

To be able to make comparisons between the temperature sensitivity of the conventional and the newly developed dual-echo method, two experiments (Experiment 2 and 3) were carried out, with identical experimental setup and comparable imaging settings. In Experiment $2(n=10)$, a spoiled gradient recalled echo sequence was employed to assess the conventional phase difference versus temperature. The procedure was repeated for Experiment $3(n=10)$ employing the newly developed dual-echo pulse sequence. 
Table 1. Imaging parameters for the different experiments. All sequences were steady state incoherent.

\begin{tabular}{llll}
\hline Parameter & Experiment 1 & Experiment 2 & Experiment 3 \\
\hline Sequence & Conv. GRE & Conv.GRE & Dual-echo GRE \\
TR $(\mathrm{msec})$ & 40 & 40 & 40 \\
te $_{1} / \mathrm{te}_{2}(\mathrm{msec})$ & $6.8 / \mathrm{NA}$ & $20 / \mathrm{NA}$ & $20.0 / 30.0$ \\
Temporal resolution $(\mathrm{sec})$ & 10 & 10 & 10 \\
Flip angle $\left({ }^{\circ}\right)$ & 20 & 20 & 60 \\
Number of excitations & 1 & 1 & 1 \\
Slice thickness $(\mathrm{mm})$ & 10 & 10 & 5 \\
Field of view $\left(\mathrm{mm}^{2}\right)$ & $480 \times 480$ & $240 \times 240$ & $240 \times 240$ \\
Bandwidth $(\mathrm{kHz})$ & 16 & 16 & 16 \\
Matrix & $256 \times 256$ & $256 \times 256$ & $256 \times 256$ \\
\hline
\end{tabular}

To assess the generalizability of referencing the temperature using the phase difference between the current pixel and neighboring pixels, a final experiment was carried out, using a homogeneous spherical phantom, positioned at the iso-center of the MR-bore. According to the theory, there should be a uniform phase over a homogeneous sphere. Two phase images were acquired, using the same pulse sequence as was used in the Experiment 3, with identical processing.

\subsection{Image Post-processing}

Images were transferred off-line from an SGI Octane (SGI, Mountain View, CA, USA) to a PC using the DICOM transfer protocol. Further processing was carried out using IDL 5.3 for Windows (Research Systems Inc, Boulder, CO, USA). Phase values are normally computed by taking the arctangent of the real and imaginary part of the MR image, yielding phase values modulo $2 \pi$. Further post-processing of the data is hampered by the resulting phase jumps (transitions from $\pi$ to $-\pi$ ). An example of a phase image is shown in Fig. 1. The central object is the heated agar gel. The surrounding objects are reliability markers to correct for main field drift.

To correctly restore the original linear phase in a pixel, its direct surrounding can be used. The solution to this problem, however, is not trivial. We unwrapped the phase images using Flynn's Minimum Discontinuity Method [15]. In addition to Flynn's algorithm, quality maps and noise filtering (of the real and imaginary part of the signal) were used to optimize the unwrapping process. Afterwards, phase maps were calculated by subtracting the reference image from consecutive phase images. From these phase maps a pixel was selected within the heated gel nearby the inserted Luxtron fiber. The local phase evolution was compared with the measurements of the Luxtron device for the times at which the echoes were acquired. For the dual-echo technique a similar procedure was followed, except that the difference was computed from the phase images that were acquired in one single acquisition. 


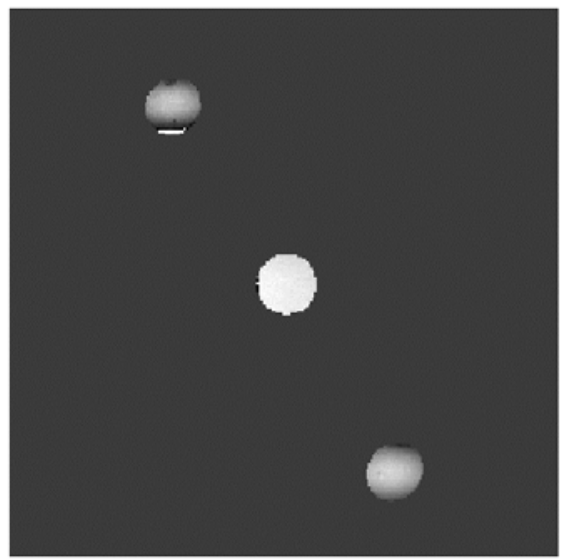

Fig. 1. A typical resulting phase image with three gels in the field of view. The outer two function as magnetic field markers. The temperature over the central gel changed during the course of the experiment. Phase of the background is masked out in this image, to improve visibility. Note the small phase jump in the upper marker.

In both experiments the mean heat coefficient was determined by linear regression. The temperature uncertainty was defined as $\pm \mathrm{SD}$ of the temperature difference between MR and the fiber optic measurements. Phase noise was estimated from the \pm $\mathrm{SD}$ of the phase signal in the non-heated reliability markers.

\section{Results}

To validate the newly developed procedure for MR thermometry, the reproducibility and accuracy of the measured temperature coefficient $\alpha$ was determined from the experiments mentioned above.

\subsection{Reproducibility of the Temperature-Dependent Screening Constant}

The temperature-dependent screening constant $\alpha$ (Table 2) was within the range of values from the literature [16], and showed low standard error for the conventional as well as the dual-echo technique. The variation of the screening constant using the conventional method was about $5 \%$, which may be caused by a variety of sources and is generally acceptable.

\subsection{Temperature Sensitivity}

The linearity and sensitivity for the dual-echo technique are depicted in Fig. 2. There was good linearity $\left(r^{2}=0.99\right)$ with temperature that holds up to at least $65^{\circ} \mathrm{C}$. 


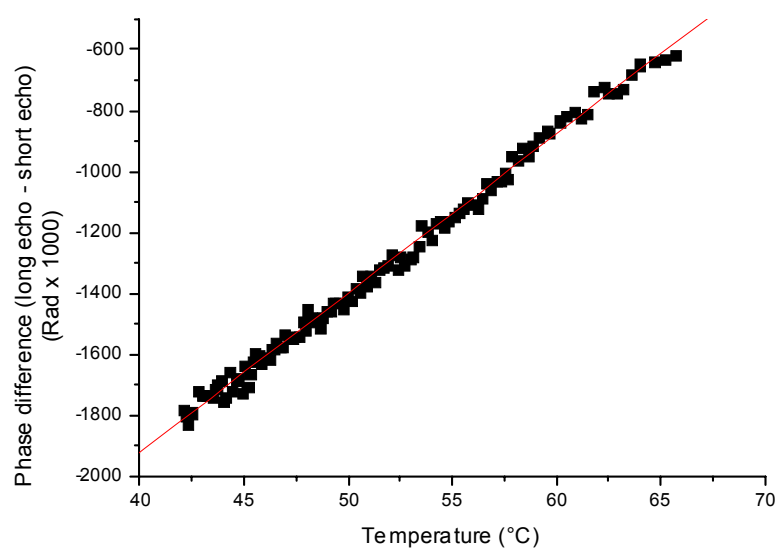

Fig. 2. The evolution of phase difference from the dual-echo technique is plotted against temperature.

The sensitivity of the dual-echo technique was slightly less than that of the conventional technique (see Table 2), as it was the result of two measurements with unrelated errors. The dual-echo sequence suffered from lower SNR due to a smaller voxel size and a relatively high flip angle.

Table 2. Temperature uncertainties, noise, and coefficients for both the conventional and the dual-echo method.

\begin{tabular}{lll}
\hline Parameter & Experiment 2 & Experiment 3 \\
\hline Sequence & Conventional GRE & Dual-echo GRE \\
MR versus Luxtron & $\pm 0.57^{\circ} \mathrm{C}$ & $\pm 1.20^{\circ} \mathrm{C}$ \\
MR noise & $\pm 1.10^{\circ} \mathrm{C}$ & $\pm 0.89^{\circ} \mathrm{C}$ \\
Temperature coefficient & $0.0106 \pm 0.0001 \mathrm{ppm} /{ }^{\circ} \mathrm{C}$ & $0.0098 \pm 0.0001 \mathrm{ppm} /{ }^{\circ} \mathrm{C}$ \\
\hline
\end{tabular}

\subsection{Spatial Stability of the Dual-Echo Phase Difference}

As seen in Fig. 3, the spatial stability of the phase was low. The dual-echo sequence used did not compensate for sampling errors during the two different readout periods. The conventional method is compensated for this type of errors, since it acquires images, which will be compared against each other, using the same gradient waveforms. Controlling and compensating for the sampling error due to the gradient waveforms of the dual-echo sequence is the topic of current research. 

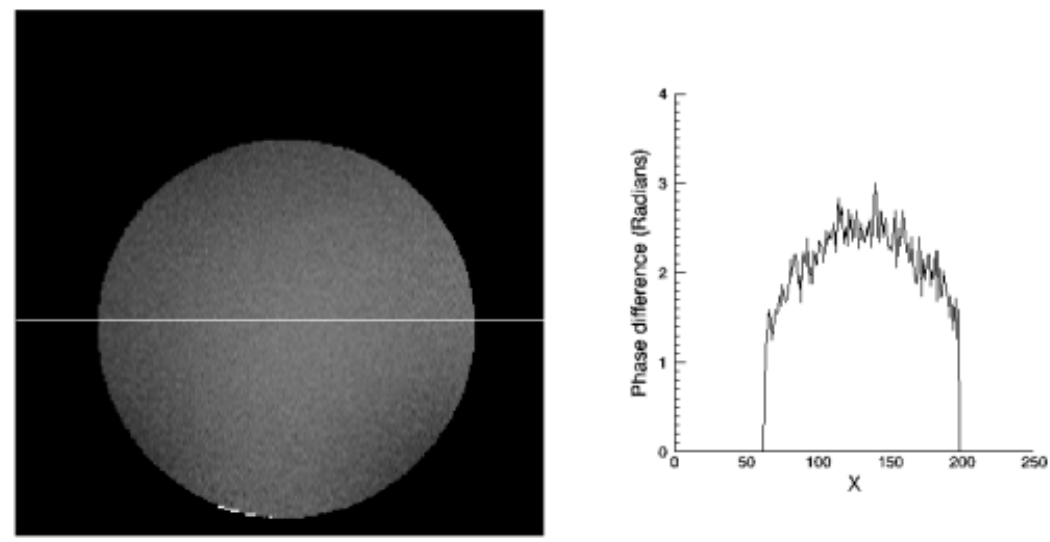

Fig. 3. Clearly visible is the change of phase value over the spherical phantom (left). In the right graph, the intensity (phase difference) profile along the horizontal line is shown. Again the background phase is masked for improved visualization. Sampling errors lead to such a pattern, and can be compensated for.

\section{Discussion}

In this paper, we have shown that the dual-echo sequence may allow for temperature encoding using the information from one MR acquisition only. Phase difference between the two generated images, varies linearly with temperature. The accuracy is slightly less when compared with the conventional thermometry method. The reproducibility of the temperature coefficient, however, was good. The inconsistency between the screening constants from the conventional method versus the dual-echo method may be explained by a slightly different makeup of the fabricated gel. Minuscule bubbles of air, may affect the evolution of phase approaching the order of the heating constant. However, in vivo micro-bubbles are not an issue. The expectation is that temperature changes can be calculated using the established screening constant of $-0.0101 \mathrm{ppm}^{\circ} \mathrm{C}^{-1}[16]$.

The found temperature resolution is sufficiently close to $1{ }^{\circ} \mathrm{C}$, and therefore within the acceptable range for hyperthermia applications. Several reports $[17,18]$ have shown a decreased sensitivity in actual in vivo settings. Consecutive experiments will include in vivo measurements to determine whether the sensitivity of the proposed dual-echo technique suffices for in vivo application.

Due to sampling error, variations in the spatially localized signal occur. These imperfections cannot be estimated from the acquired images. Theoretically they can be assessed when using a slightly adapted version of the dual-echo sequence, incorporating refocusing pulses. Removing these imperfections is the strategy to overcome the spatial limitation of the dual-echo technique. In order to allow quantification of temperature from pixels relative to others in the same image, the 
overall phase should be constant. Using this constant, one can normalize the phase to a properly referenced temperature.

\section{References}

1. Sherar MD, Moriarty JA, Kolios MC, et al. Comparison of thermal damage calculated using magnetic resonance thermometry, with magnetic resonance imaging post-treatment and histology, after interstitial microwave thermal therapy of rabbit brain. Phys Med Biol 2000; 45:3563-76.

2. Gertner MR, Worthington AE, Wilson BC, Sherar MD. Ultrasound imaging of thermal therapy in in vitro liver. Ultrasound Med Biol 1998; 24:1023-32.

3. McDannold NJ, Jolesz FA. Magnetic resonance image-guided thermal ablations. Top Magn Reson Imaging 2000; 11:191-202.

4. Quesson B, de Zwart JA, Moonen CT. Magnetic resonance temperature imaging for guidance of thermotherapy. J Magn Reson Imaging 2000; 12:525-33.

5. Madio DP, van Gelderen P, DesPres D, et al. On the feasibility of MRI-guided focused ultrasound for local induction of gene expression. J Magn Reson Imaging 1998; 8:101-4.

6. de Zwart JA, Salomir R, Vimeux F, Klaveness J, Moonen CTW. On The Feasibility Of Local Drug Delivery Using Thermo-Sensitive Liposomes and MR-Guided Focused Ultrasound. Proceedings of ISMRM 2000.

7. Sapareto SA, Dewey WC. Thermal dose determination in cancer therapy. Int J Radiat Oncol Biol Phys 1984; 10:787-800.

8. Bottomley PA, Foster TH, Argersinger RE, Pfeifer LM. A review of normal tissue hydrogen NMR relaxation times and relaxation mechanisms from 1-100 MHz: dependence on tissue type, NMR frequency, temperature, species, excision, and age. Med Phys 1984; 11:425-48.

9. Hindman JC. Proton resonance shift of water in gas and liquid states. J Chem Phys 1966; 44:4582-92.

10. Nelson TR, Tung SM. Temperature dependence of proton relaxation times in vitro. Magn Reson Imaging 1987; 5:189-99.

11. Schneider WG, Bernstein HJ, Pople JA. Proton magnetic resonance chemical shift of free (gaseous) and associated (liquid) hydride molecules. J Chem Phys 1985; 28:601-7.

12. Ishihara $\mathrm{Y}$, Calderon $\mathrm{A}$, Watanabe $\mathrm{H}$, et al. A precise and fast temperature mapping using water proton chemical shift. Magn Reson Med 1995; 34:814-23

13. Salomir R, Palussiere J, Vimeux FC, et al. Local hyperthermia with MR-guided focused ultrasound: spiral trajectory of the focal point optimized for temperature uniformity in the target region. J Magn Reson Imaging 2000; 12:571-83.

14. de Poorter J, Noninvasive MRI thermometry with the proton resonance frequency method: study of susceptibility effects. Magn Reson Med 1995; 34:359-67.

15. Ghiglia DC, Pritt MD. Two-dimensional Phase Unwrapping: Theory, Algorithms, and Software: John Wiley and Sons, Inc., 1998.

16. Peters RD, Hinks RS, Henkelman RM. Ex vivo tissue-type independence in protonresonance frequency shift MR thermometry. Magn Reson Med 1998; 40:454-9.

17. De Poorter J, De Wagter C, De Deene Y, et al.. Noninvasive MRI thermometry with the proton resonance frequency (PRF) method: in vivo results in human muscle. Magn Reson Med 1995; 33:74-81.

18. Botnar RM, Steiner P, Dubno B, et al. Temperature quantification using the proton frequency shift technique: In vitro and in vivo validation in an open 0.5 Tesla interventional MR scanner during RF ablation. J Magn Reson Imaging 2001; 13:437-44. 